Check for updates

Cite this: J. Mater. Chem. C, 2017, 5, 12430

Received 15th August 2017, Accepted 18th October 2017

DOI: $10.1039 / c 7 t c 03697 c$

rsc.li/materials-c

\title{
3D-printed barium titanate/poly-(vinylidene fluoride) nano-hybrids with anisotropic dielectric properties
}

\author{
N. Phatharapeetranun, (D) ${ }^{\text {ab }}$ B. Ksapabutr, ${ }^{b}$ D. Marani, (D) ${ }^{c}$ J. R. Bowen (D) and \\ V. Esposito iD *a
}

\begin{abstract}
Electrospun $\mathrm{BaTiO}_{3}$ nanofibers (BTNFs) are synthesized and blended in a poly(vinylidene fluoride) (PVDF) matrix to obtain a flexible nano-hybrid composite with high dielectric constant (flexible high- $k$ ). The blending is performed with different BTNF contents $(0.6,4.5,20$ vol\%). The rheological properties of the starting materials are optimized to shape the hybrid by the precision-extrusion-based fuse deposition modeling technique. The 3D-printed BTNFs allow complex shapes with different degrees of fiber alignment as the result of printing shear stress and the chemical composition of the starting material. The dielectric properties of the nano-hybrid are controlled by anisotropy with an enhancement in the nanofiber cross direction ( $\perp$ ), where the dielectric constant $k^{\perp}$ at $1 \mathrm{kHz}$ is increased to ca. 200 from 13 of the PVDF matrix.
\end{abstract}

\section{Introduction}

Ferroelectric ceramic-polymer nanohybrid materials play a crucial role in flexible electronics and in energy technologies (e.g. capacitors, sensors, and transducers), due to their merits of enabling the tailoring of specific dielectric, thermal, and mechanical properties and easy processing..$^{1-4}$ By integrating the advantages of the two phases, nanohybrid materials can offer enhanced performances with respect to those of the individual components. ${ }^{5}$ The performance of this kind of material generally depends both on the individual properties of the two components, and on the nature and extension of the hybrid interface. ${ }^{6,7}$ This latter feature can easily be modulated by varying the mixing length scale and the nature of the polymer-ceramic interactions. Indeed, the larger the interfacial phase, the greater the influence of the ceramic component on the original characteristics of the PVDF. ${ }^{7}$ This confers tunable properties that make hybrid materials highly appealing for a wide range of applications. ${ }^{7}$ Yet, the unlimited possibilities derived from this approach pave the way for advanced new materials with unique properties. ${ }^{7}$

Owing to its high dielectric constant $(k)$, high mechanical strength, excellent chemical stability, non-toxicity, and easy

\footnotetext{
${ }^{a}$ DTU Energy, Technical University of Denmark, Risø Campus, Frederiksborgvej 399, DK-400o, Roskilde, Denmark.E-mail: vies@dtu.dk

${ }^{b}$ Department of Materials Science and Engineering, Faculty of Engineering and Industrial Technology, Silpakorn University, Nakornpathom, Thailand

${ }^{c}$ Centro de Engenharia, Modelagem e Ciências Sociais Aplicadas, Universidade Federal do ABC Av. dos Estados 5001, Santo André (SP), 09210-580, Brazil
}

processability, the electroactive poly(vinylidene fluoride) (PVDF) is the most used polymer in the dielectric materials field. ${ }^{8,9}$ Concerning the ceramic component, among others barium titanate $\left(\mathrm{BaTiO}_{3}: \mathrm{BT}\right)$, a lead-free high- $k$ ferroelectric ceramic material with a perovskite structure, is used in many applications due to its attractive piezoelectric and dielectric properties. ${ }^{10,11}$

Given their exceptional properties and non-toxicity, hybrid systems based on PVDF-BT combinations have been largely investigated as dielectric materials. ${ }^{12,13}$ Nanostructured functional fillers with a high aspect ratio (e.g. one-dimensional, 1D) have been widely used with the aim of increasing the hybrid interface. ${ }^{14-20}$ A significant effect on the dielectric properties of PVDF has been obtained. ${ }^{13-16}$ Among others, nanofibers, prepared by electrospinning, offer the further advantage of easy control over the morphology and the compositional features. ${ }^{18-20}$

Several shaping methods have been traditionally used to process hybrid systems into thin film. ${ }^{21-23}$ Currently, there is significant development in three dimensional (3D) assembly technology, namely 3D printing. One of the most interesting 3D printing techniques is computer-controlled fabrication by solid freeform, also known as fuse deposition modeling (FDM). This technique produces objects with controlled complex shapes and reduced size with short processing times, with relatively low cost equipment. ${ }^{24}$ Recently, Castles et al. have demonstrated $\mathrm{BaTiO}_{3}$ powder/ABS polymer dielectric composites by FDM. ${ }^{25}$ They obtained a dielectric constant value for the material at $20 \mathrm{vol} \%$ of BT of 6.24 , which matched those of bulk unprinted materials. Sun et al. prepared lithium ion micro-batteries by $\mathrm{FDM}^{26}$ that exhibited high energy and power density. 
Furthermore, when anisotropic fillers are used, their orientation within the polymeric matrix is an additional parameter that has been demonstrated to significantly enhance the performances of the BTNFs/PVDF-based systems. ${ }^{27}$

In the present work, we propose a novel approach for preparing anisotropic BTNFs/PVDF 3D-nanohybrid dielectric materials. The nanohybrids are designed and fabricated by FDM, where the nanofibers are embedded and aligned in the PVDF matrix by tunable internal friction during the extrusion. We especially analyze phase compositions and properties in the hybrid as a result of the rheological properties by varying the BTNF loading and the extrusion forces that lead to fiber alignment during the $3 \mathrm{D}$ printing. The overall aim is thus to tune the dielectric properties in the $3 \mathrm{D}$ range by creating a "3D-printed" anisotropy in the BTNFs/PVDF hybrid material. This consists in combining short-range orientation of the nanofibers, at the microstructural level, with long-range directionality in the complex shape by the $3 \mathrm{D}$ printing technique.

\section{Experimental}

\subsection{Materials}

The starting precursors used in this work included barium acetate (Sigma-Aldrich, $\mathrm{Ba}\left(\mathrm{CH}_{3} \mathrm{COO}\right)_{2}$ ), acetic acid (SigmaAldrich, $\mathrm{CH}_{3} \mathrm{COOH}$ ), titanium isopropoxide (Sigma-Aldrich, $\left.\mathrm{Ti}\left(\mathrm{OCH}\left(\mathrm{CH}_{3}\right)_{2}\right)_{4}\right)$, polyvinylpyrrolidone (Sigma-Aldrich, PVP $\left(M_{\mathrm{w}} \approx 1300000\right)$ ), ethanol (Sigma-Aldrich, $\left.\mathrm{C}_{2} \mathrm{H}_{5} \mathrm{OH}\right)$, and $\mathrm{N}$-methyl-2-pyrrolidone (Sigma-Aldrich, NMP, $\mathrm{C}_{5} \mathrm{H}_{9} \mathrm{NO}$ ). All the chemicals were analytical reagent grade and used without further purification.

\subsection{Electrospinning of $\mathrm{BaTiO}_{3}$ nanofibers (BTNFs)}

For the BTNF synthesis, ${ }^{28} 1.275 \mathrm{~g}$ of $\mathrm{Ba}\left(\mathrm{CH}_{3} \mathrm{COO}\right)_{2}$ was dissolved in $3 \mathrm{~mL}$ of $\mathrm{CH}_{3} \mathrm{COOH}$ for $2 \mathrm{~h}$ at room temperature, under argon flow. Then, $1.475 \mathrm{~mL}$ of $\mathrm{Ti}\left(\mathrm{OCH}\left(\mathrm{CH}_{3}\right)_{2}\right)_{4}$ was added dropwise into the solution under magnetic stirring. The precursor mixture was stirred for $2 \mathrm{~h}$ to achieve complete dissolution and mixing. A solution of $0.2 \mathrm{~g}$ of PVP dissolved in $3 \mathrm{~mL}$ of $\mathrm{C}_{2} \mathrm{H}_{5} \mathrm{OH}$ was added to the ceramic precursor. The precursor mixture was loaded into a plastic syringe equipped with a metallic needle tip. Then, $15 \mathrm{kV}$ was applied to the precursor and the distance between the tip-end and collector was fixed at $9.0 \mathrm{~cm}$. All the electrospinning experiments were carried out by an electrospinning machine (RT Advanced, Linari engineering, Italy) at room temperature. The as-synthesized fibers were dried in a fume-hood at room temperature for $24 \mathrm{~h}$ to remove the humidity.

In order to obtain crystallized BT nanofibers (BTNFs), the calcination temperature for the electrospun fibers was evaluated with the aid of TG/DTA (Netzsch STA 409C/CD, Germany) and XRD (XRD: Bruker D8 Robot Tools X-ray diffraction, Bruker, Germany). The TG/DTA analysis was performed in air flux from room temperature to $850{ }^{\circ} \mathrm{C}$ at a heating rate of $1{ }^{\circ} \mathrm{C} \min ^{-1}$.
The XRD analysis was carried out ex situ with a scan speed of $0.6^{\circ} 2 \theta \mathrm{min}^{-1}$ at a $2 \theta$ range of $20^{\circ}$ to $80^{\circ}$ on samples calcined at $400,500,600,700$, and $800{ }^{\circ} \mathrm{C}$ for $1 \mathrm{~h}$. The diameter and the morphology of the crystalline fibers were evaluated with a field emission gun scanning electron microscope (ZEISS Merlin, Carl Zeiss Microscopy $\mathrm{GmbH}$, Germany) at an accelerating voltage of $10 \mathrm{kV}$ with an In-lens and SE1 electron detector.

\subsection{Preparation of anisotropic BTNFs/PVDF 3D-nanohybrid dielectric materials}

Anisotropic BTNFs/PVDF 3D-nanohybrid dielectric materials were fabricated by FDM. The concentration of PVDF in the NMP solution was fixed at $30 \mathrm{wt} \%$ to match with the viscosity range of thick paste materials (20000-70000 $\mathrm{mPa}$ s) for consumer grade 3D printers (Zmorph 2.0S, ZMorph Sp. z o.o., Poland). First, $30 \mathrm{~g}$ of PVDF was dissolved in $100 \mathrm{~g}$ of NMP. The solution was then stirred at $50{ }^{\circ} \mathrm{C}$ with constant stirring for $2 \mathrm{~h}$ until a homogeneous solution appeared. The loading of the BTNFs was varied from 0 (pure PVDF) to 20 vol\% to cover the range of concentrations allowed by the printer used. Then, the defined compositions of the BTNFs and the PVDF solution were stirred with constant rate. The resulting mixture was subjected to ultrasonic agitation at a frequency of about $24 \mathrm{kHz}$ for 30 minutes. The resulting solution was transferred to an extrusion syringe with different nozzles of diameters $2.5 \mathrm{~mm}$ and $1.2 \mathrm{~mm}$ and then was printed by a $3 \mathrm{D}$ printer. All the samples were printed at room temperature without using a hot bed.

The rheology characteristics of pure PVDF and the BTNFs/ PVDF nanohybrid materials before printing were evaluated by a rotational rheometer (MCR viscometer 302, Anton Paar, Austria). The solutions were characterized in rotational mode. A constant temperature of $21{ }^{\circ} \mathrm{C}$ was maintained during the experiments using a temperature control unit. All the experiments were performed using a cone-parallel spindle of $25 \mathrm{~mm}$ in diameter and $4^{\circ}$ angle (CP25-4). To remove any effects due to the sampling and loadings, the measurements were performed using a pre-shear at $1 \mathrm{~s}^{-1}$ for 1 minute followed by 1 minute at rest ( $0 \mathrm{~s}^{-1}$ shear rate), and again at $1 \mathrm{~s}^{-1}$ for 1 minute. The experiments were conducted in the shear rate of $1-100 \mathrm{~s}^{-1}$ in up and down ramp.

The phase compositions were analyzed by the XRD technique (XRD: Bruker D8 Robot Tools X-ray diffraction, Bruker, Germany). The XRD analysis was carried out with a scan speed of $0.6^{\circ} 2 \theta \mathrm{min}^{-1}$ at a $2 \theta$ range of $10^{\circ}$ to $50^{\circ}$.

The surface morphology and fiber alignment of the 3D-nanohybrid materials were evaluated with a scanning electron microscope (TM3000 Tabletop Scanning Electron Microscope, Hitachi, Japan) at an accelerating voltage of $15 \mathrm{kV}$ with a backscattered electron detector.

The degree of fiber orientation in three dimensions was evaluated by the focused ion beam (FIB) SEM serial sectioning technique by simultaneously collecting In-lens (on beam axis SE1) and SE2 (Everhart-Thornley) detector secondary electron (SE) images (Zeiss 1540 XB Crossbeam Scanning Electron Microscope, Carl Zeiss Microscopy $\mathrm{GmbH}$, Germany). The reconstructed volume had dimensions of approximately $20 \times 17 \times 20 \mu \mathrm{m}$, with 
the longest axis perpendicular to the extrusion direction and parallel to the FIB slicing direction. The voxel dimensions were $39.5 \mathrm{~nm}$ in the SEM imaging plane with a slice thickness of $60.6 \mathrm{~nm}$. The dual imaging mode raw data were global intensity gradient corrected, aligned, and cropped. ${ }^{29,30}$ All image processing was performed using an in-house image analysis code written in MATLAB. The BTNFs were segmented in 3D from the PVDF matrix using a combination of image intensity and intensity gradient in both the In-lens and SE2 image modes combined with visual inspection to achieve the optimum segmentation and minimize image artifacts due to the serial sectioning technique. The segmented BTNF phase was skeletonized using a parallel medial axis thinning approach ${ }^{31,32}$ to determine the centerlines of the individual fibers, which were then converted to a network graph of links and nodes to characterize the length and orientation of fiber fragments. ${ }^{33}$ Principle component analysis (PCA) using inbuilt MATLAB functions was then performed on the 3D coordinates of the centerlines of each fiber fragment to determine the orientation of the longest axis of the coordinate voxel point cloud in terms of azimuthal and elevation angles relative to the extrusion direction.

\subsection{Dielectric property measurement}

Broadband dielectric measurements of both pure PVDF and BTNFs/ PVDF 3D-nanohybrid dielectric materials for different vol\% of BTNFs were carried out by using an Impedance/Gain-Phase Analyzer (Solartron 1260A, Solartron Analytical, UK). All measurements were carried out in air at room temperature, in the frequency range of $100 \mathrm{~Hz}$ to $1 \mathrm{MHz}$ with an applied biasing voltage of $0.2 \mathrm{~V}$. The frequency dependence of the dielectric constant $(k)$ at room temperature can be calculated from the following relation. ${ }^{34}$

$$
k=\frac{C_{\mathrm{p}} \times t}{A \times \varepsilon_{0}}
$$

where, $C_{\mathrm{p}}$ is the capacitance of the materials $(\mathrm{F}), t$ is the thickness of the nanohybrid dielectric material (m), $A$ is the area of the electrode $\left(\mathrm{m}^{2}\right)$, and $\varepsilon_{0}$ is the vacuum permittivity having a value of $8.854 \times 10^{-12} \mathrm{~F} \mathrm{~m}^{-1}$.

Dielectric properties were measured in both the extrusion direction (parallel, $k^{\|}$) and transverse direction (cross, $k^{\perp}$ ).

\subsection{Methods}

The observed enhancement in the dielectric properties of the developed hybrids was discussed according to the effective medium theory (EMT) and rule of mixture (ROM) models.

The EMT model, developed by Rao et al., ${ }^{35}$ enables the estimation of the dielectric properties of composite systems by including the effect of the morphology and size of the ceramic fillers. The model is described by the following equation:

$$
\varepsilon=\varepsilon_{\mathrm{m}}\left[1+\frac{f\left(\varepsilon_{\mathrm{f}}-\varepsilon_{\mathrm{m}}\right)}{\varepsilon_{\mathrm{m}}+n(1-f)\left(\varepsilon_{\mathrm{f}}-\varepsilon_{\mathrm{m}}\right)}\right]
$$

where $n$ is the shape-dependent parameter, $f$ is the volume fraction of the ceramic fillers, and $\varepsilon, \varepsilon_{\mathrm{f}}$ and $\varepsilon_{\mathrm{m}}$ represent the dielectric constant of the nanocomposites, ceramic, and polymer matrix, respectively.
The ROM model instead includes in the estimation the shape fillers. The dielectric constant is estimated from the following relation. ${ }^{36}$

$$
\varepsilon_{\text {mix }}=\varepsilon_{\mathrm{m}}+\left[\frac{\left(\varepsilon_{\mathrm{i}}-\varepsilon_{\mathrm{m}}\right)\left(\varepsilon_{\mathrm{i}}+5 \varepsilon_{\mathrm{m}}\right)}{3\left(\varepsilon_{\mathrm{i}}+\varepsilon_{\mathrm{m}}\right)}\right] V_{\mathrm{i}}
$$

where $\varepsilon_{\text {mix }}, \varepsilon_{\mathrm{i}}, \varepsilon_{\mathrm{m}}$ are the dielectric constants of the composite, ceramic filler material, and matrix material, respectively. $V_{\mathrm{i}}$ is the volume fraction of ceramic fillers.

\section{Results and discussion}

For the formation of the BTNFs, crystallization and microstructures are controlled by the sol-gel chemistry, the electrospinning, and the calcination conditions. For the chemistry and the electrospinning conditions, previous work by Junhan et al. was used as a reference, while the degree of crystallinity can be modulated by the calcination thermal treatment. ${ }^{28}$ To optimize the heat-treatment process, TG/DTA analysis was carried out on the as-synthesized fibers. The thermal decomposition behavior of the as-synthesized fibers is shown in Fig. 1. About $10 \%$ weight loss from 80 to $150{ }^{\circ} \mathrm{C}$ was related to the evaporation of residual solvent. The weight loss between $150{ }^{\circ} \mathrm{C}$ and $330{ }^{\circ} \mathrm{C}$ and a sharp exothermic peak at $280{ }^{\circ} \mathrm{C}$ were correlated to the decomposition of organic precursors. ${ }^{24,37}$ The weight loss (about 7\%) and the corresponding broad exothermic peak observed between $380{ }^{\circ} \mathrm{C}$ and $550{ }^{\circ} \mathrm{C}$ are consistent with the decomposition of organic groups from the organometallic precursor and its intermediate phases, $\mathrm{BaCO}_{3} \cdot{ }^{38}$ The weight loss between $570{ }^{\circ} \mathrm{C}$ and $700{ }^{\circ} \mathrm{C}$ is indicative of the onset of crystallization of BT nanofibers. The TG/DTA results reveal about $50 \%$ weight loss that was observed with no further weight loss at temperatures $>700{ }^{\circ} \mathrm{C}$. Accordingly, the synthesized fibers were completely crystallized and intermediate phases were removed at temperatures over $700{ }^{\circ} \mathrm{C} .{ }^{38,39}$

The XRD patterns of the as-synthesized and calcined fibers at temperatures between $400{ }^{\circ} \mathrm{C}$ and $800{ }^{\circ} \mathrm{C}$ are presented in Fig. 2 . The as-synthesized fibers were amorphous consisting of un-oriented structures. $^{28,39-41}$ Two peaks were observed at $2 \theta$ around $27^{\circ}$ and $43^{\circ}$

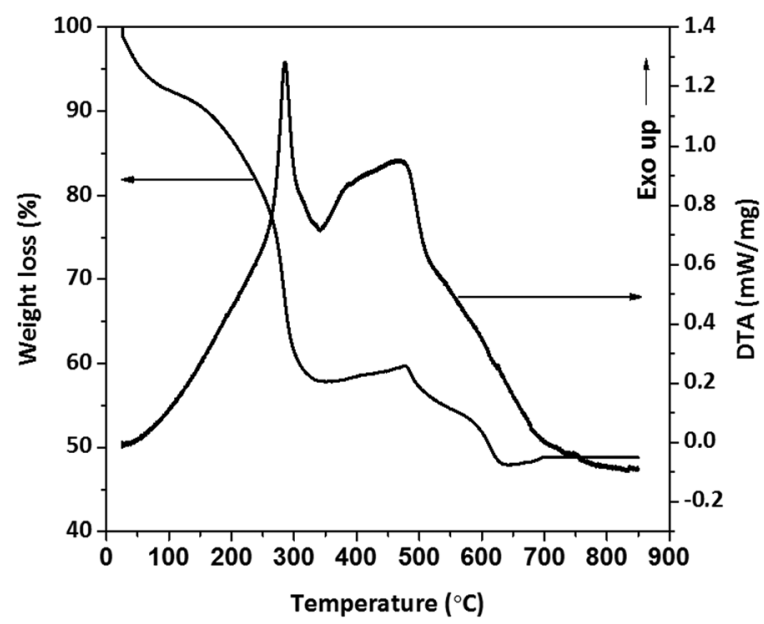

Fig. 1 TG/DTA profiles of the as-synthesized fibers. 


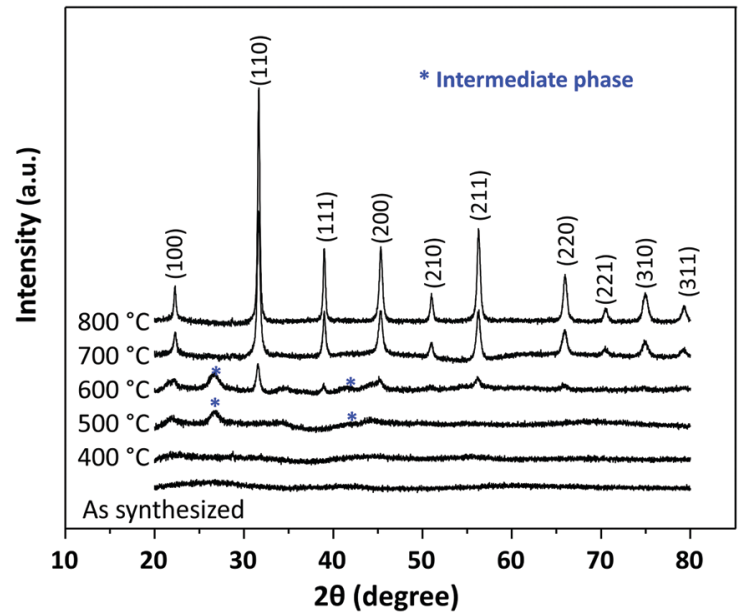

Fig. 2 X-ray diffraction patterns of the as-synthesized fibers and BTNFs calcined for $1 \mathrm{~h}$ at different temperatures.

for the samples treated at $500{ }^{\circ} \mathrm{C}$ and $600{ }^{\circ} \mathrm{C}$, which are consistent with $\mathrm{BaTi}_{2} \mathrm{O}_{4}$ and $\mathrm{BaCO}_{3}{ }^{24}$ The calcined sample at $600{ }^{\circ} \mathrm{C}$ exhibited the onset of BT perovskite crystallization together with the nonperovskite peaks of an intermediate phase, $\mathrm{BaCO}_{3} \cdot{ }^{28,40}$ Well-defined perovskite $\mathrm{BaTiO}_{3}$ patterns were instead observed for the samples treated at $700{ }^{\circ} \mathrm{C}$, and the disappearance of the non-perovskite peaks is indicative of the decomposition of $\mathrm{BaCO}_{3}$. At $800{ }^{\circ} \mathrm{C}$, the perovskite peak pattern became marked, due to the complete reaction of the BT formation.

The morphologies of the as-synthesized and calcined fibers were then investigated. Fig. 3 shows the morphologies of the as-synthesized fibers and BTNFs after calcination at $800{ }^{\circ} \mathrm{C}$. The as-synthesized nanofibers (Fig. $3 \mathrm{a}$ and b) exhibit smooth and uniform surfaces with a random fiber orientation, whereas a polycrystalline surface is observed for the nanofibers calcined at $800{ }^{\circ} \mathrm{C}$ (Fig. 3c). The crystalline nanofibers are fragile and break easily in agreement with the literature. ${ }^{41-44}$ The nanofiber diameter is observed to decrease from $800 \mathrm{~nm}$ to $200 \mathrm{~nm}$ after calcination due to the decomposition of the organic components and the crystallization of the phase of interest. ${ }^{28,45}$ Upon the calcination process, the diameter is reduced by $75 \%$.

There have been no previous reports on the preparation of PVDF composites using a consumer 3D printer with different nozzle diameters. The X-ray diffraction (XRD) pattern was then used to investigate the change in crystallinity of the 3D printed materials from $2.5 \mathrm{~mm}$ and $1.2 \mathrm{~mm}$ of nozzle diameter due to the addition of BTNFs in the PVDF matrix.

The XRD pattern of pure PVDF in Fig. 4 shows three main characteristic peaks; a sharp peak at 20.01 corresponds to the $\beta$-phase (110) crystal with a fully stretched all-trans planar zigzag conformation and indicates the semi-crystalline nature of PVDF. ${ }^{34,46-49}$ Furthermore, the peaks at 18.41 and 39.21 which are assigned to the $\alpha$-phase characteristic of PVDF were observed. ${ }^{47-49}$ In the XRD pattern of the BTNFs/PVDF nanohybrids crystalline peaks, corresponding to the reflection planes at (100), (110), (111), and (200) of the perovskite BT structures are also identified. Interestingly, with an increasing
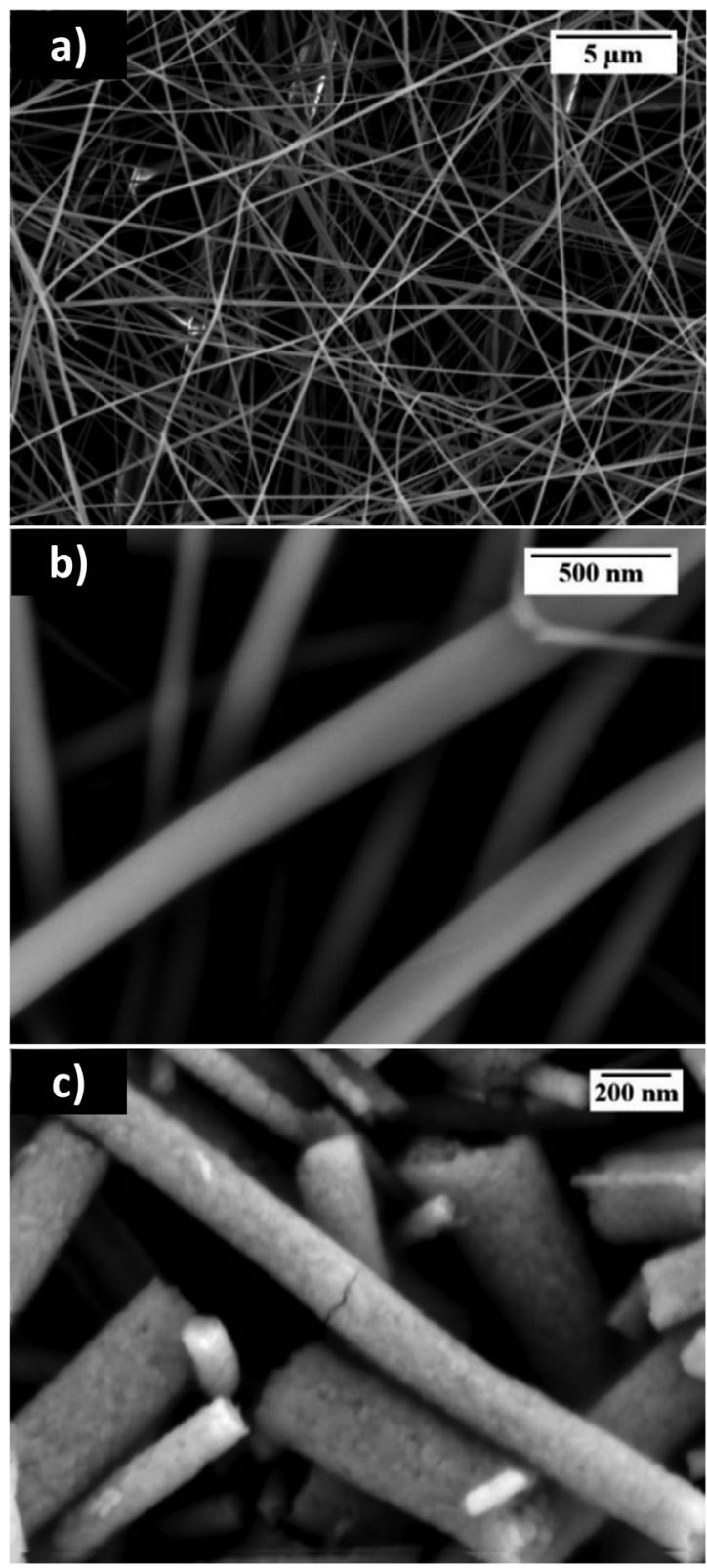

Fig. 3 FE-SEM In-lens SE1 images of (a) and (b) as-synthesized fibers and (c) BTNFs calcined at $800^{\circ} \mathrm{C}$ for $1 \mathrm{~h}$.

amount of BTNFs from $0.6 \mathrm{vol} \%$ to $4.5 \mathrm{vol} \%$, the peak intensity of the $\alpha$ and $\beta$ crystalline phases increases with respect to the pure PVDF, indicating that the addition of a small amount of BTNFs can promote a more regular arrangement for PVDF, and the added BTNFs act as nuclei for PVDF crystallization. When the loading of $20 \mathrm{vol} \%$ is reached, the intensity of the $\alpha$ and $\beta$ characteristic peaks decreased, especially the $\alpha$ characteristic peak at 39.21. At this content of BTNFs, the $\beta$ phase is dominant. The addition of a large amount of BTNFs might destroy the molecular regularity of the PVDF chain. ${ }^{47}$ In addition, there is no significant difference in the intensity of the $\beta$-phase of the obtained hybrids from $2.5 \mathrm{~mm}$ and $1.2 \mathrm{~mm}$ of nozzle diameter (line (4d) and (4e)), respectively, indicating that 


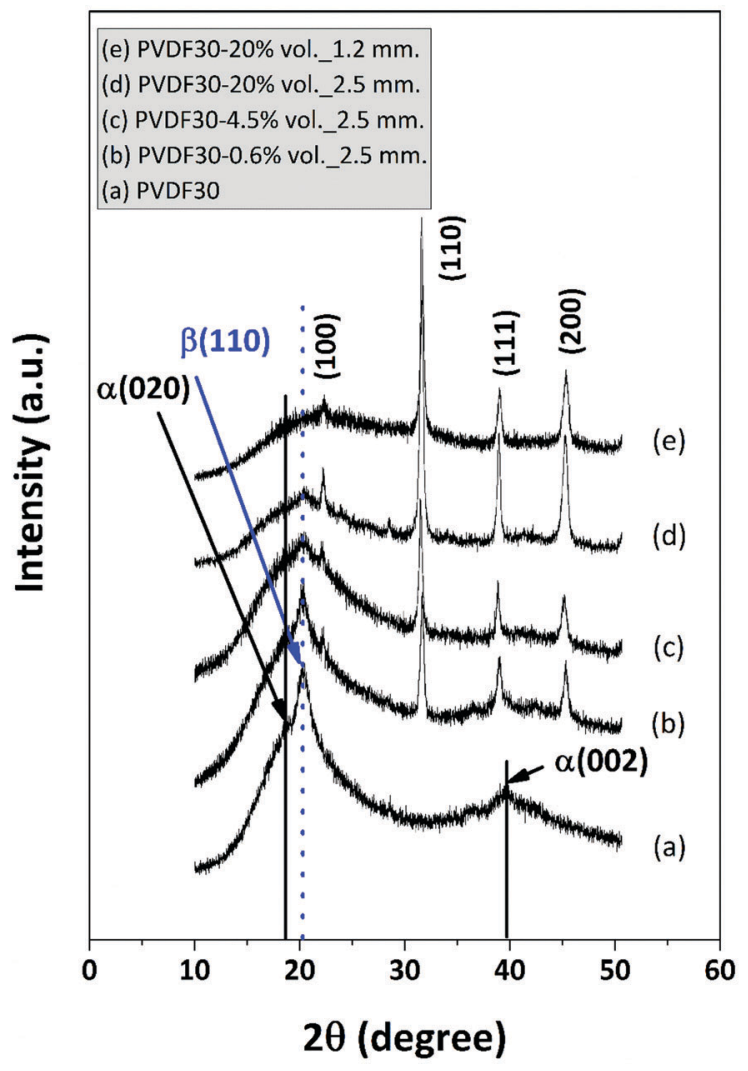

Fig. 4 X-ray diffraction patterns of the BTNFs/PVDF 3D-nanohybrid materials at different vol\% of BTNFs.

the nozzle diameter has no effect on the $\beta$ phase crystallinity of the PVDF hybrids.

For the FDM technique, the maximum solid loading of the filler in the polymer matrix that enables printing is $\sim 50 \mathrm{wt} \%{ }^{25,26,50}$ Indeed, at high content of filler, the shear stress at a nozzle extruder is high, resulting in the unstable flow at the extrusion nozzle. ${ }^{50}$ In this work, we used a consumer grade $3 \mathrm{D}$ printer (Zmorph 2.0S) to fabricate 3D-printed dielectric materials as shown in Fig. 5a. The loading of BTNFs is varied from $0.6 \mathrm{vol} \%$ to $20 \mathrm{vol} \%$, and nozzle extruders of $2.5 \mathrm{~mm}$ and $1.2 \mathrm{~mm}$ were used to explore the effect of processing conditions on the nanofiber alignment. With a $2.5 \mathrm{~mm}$ nozzle diameter, a random distribution of the nanofibers is observed in the SEM images for the sample at 0.6 vol\% of BTNFs (Fig. 5b), whereas with increasing content the BT nanofibers show clear alignment in the printing direction (Fig. 5d). By contrast, in the hybrid at 20 vol\% prepared using the nozzle $1.2 \mathrm{~mm}$ in diameter (Fig. 5e) the BT nanofibers are randomly distributed into the polymer matrix. This can be ascribed to an unstable flow generated from too high shear stress. ${ }^{50}$ From the obtained results, we could produce a 3Dprinted object as a complex shape and controllable fiber alignment in the polymer matrix by this technique.

To further support the 2D observations in Fig. 5, FIB-SEM 3D reconstruction of the $20 \mathrm{vol} \% \mathrm{BTNF} / \mathrm{PVDF}$ nanohybrid sample was evaluated to verify and semi-quantitatively evaluate the degree of 3D fiber orientation. 3D FIB-SEM investigations
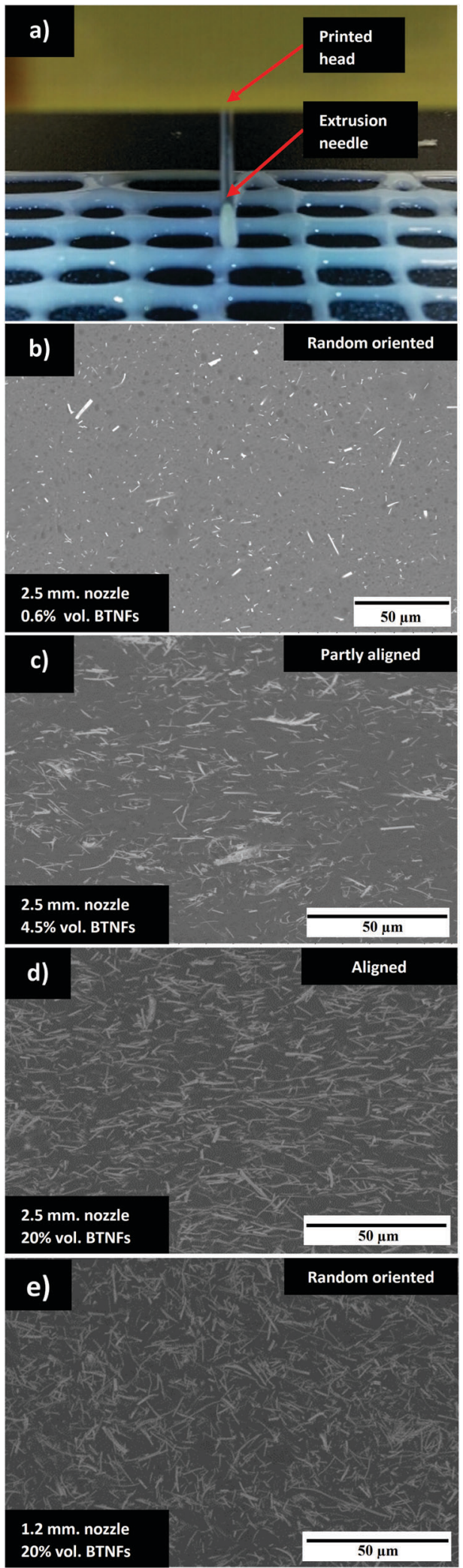

Fig. 5 (a) Extrusion-based FDM technique for BTNFs-PVDF dielectric materials; fiber alignment of BTNFs/PVDF materials printed with a $2.5 \mathrm{~mm}$ nozzle diameter: (b) 0.6 vol\% BTNFs, (c) 4.5 vol\% BTNFs, and (d) 20 vol\% BTNFs; and (e) 20 vol\% BTNFs printed with a $1.2 \mathrm{~mm}$ nozzle diameter. 
inherently sample a smaller amount of representative microstructure. From the 2D images it can be seen that the microstructure contains $n m-\mu \mathrm{m}$ sized highly elongated features and is spatially heterogeneous requiring investigation of large volumes. For 3D reconstructions, these are conflicting requirements in terms of data acquisition time. Fig. 6a shows a representative slice of the volume analyzed. Here, it can be seen in comparison with Fig. 5d that the volume captures a visually representative volume with sufficient spatial resolution. Fig. 6a contains both the image modes, which are useful to distinguish internal porosity from edge effects and minimize FIB milling artifacts during segmentation. Visual inspection of the volume reveals internal porosity of both the BTNFs and the PVDF that is not visible in the polished cross-sections in Fig. 5. In some reconstructed volume locations porosity-induced artifacts were created in the segmentation and thus add uncertainty to quantitative analysis. However, of the available quantitative parameters from the $3 \mathrm{D}$ analysis, fiber orientation is one of the least sensitive to segmentation errors compared with volume fraction or particle size distribution determination. Thus, in the following analysis we discuss the results in a semi-quantitative manner.

Fig. 6b shows the discretized skeleton of the BTNFs. Inspection of the BTNF phase after image segmentation revealed that although most of the BTNFs appear as isolated fibers in $2 \mathrm{D}$, in $3 \mathrm{D}$ the majority of fibers are in contact. Thus after the reconstruction from an image analysis point of view the majority of the BTNFs in the $20 \mathrm{vol} \%$ sample appear as an interconnected network. Thus breaking the skeleton into a network graph of links and nodes was necessary to assess the orientation distribution of individual fiber fragments (links) visualized in arbitrary colors in Fig. 6b. The orientation of each fiber fragment, as shown on the unit sphere in Fig. 6c, was determined by standard PCA where the direction of the largest variance in voxel coordinates determines the primary axis of fiber orientation. The points on the sphere represent a single fragment and
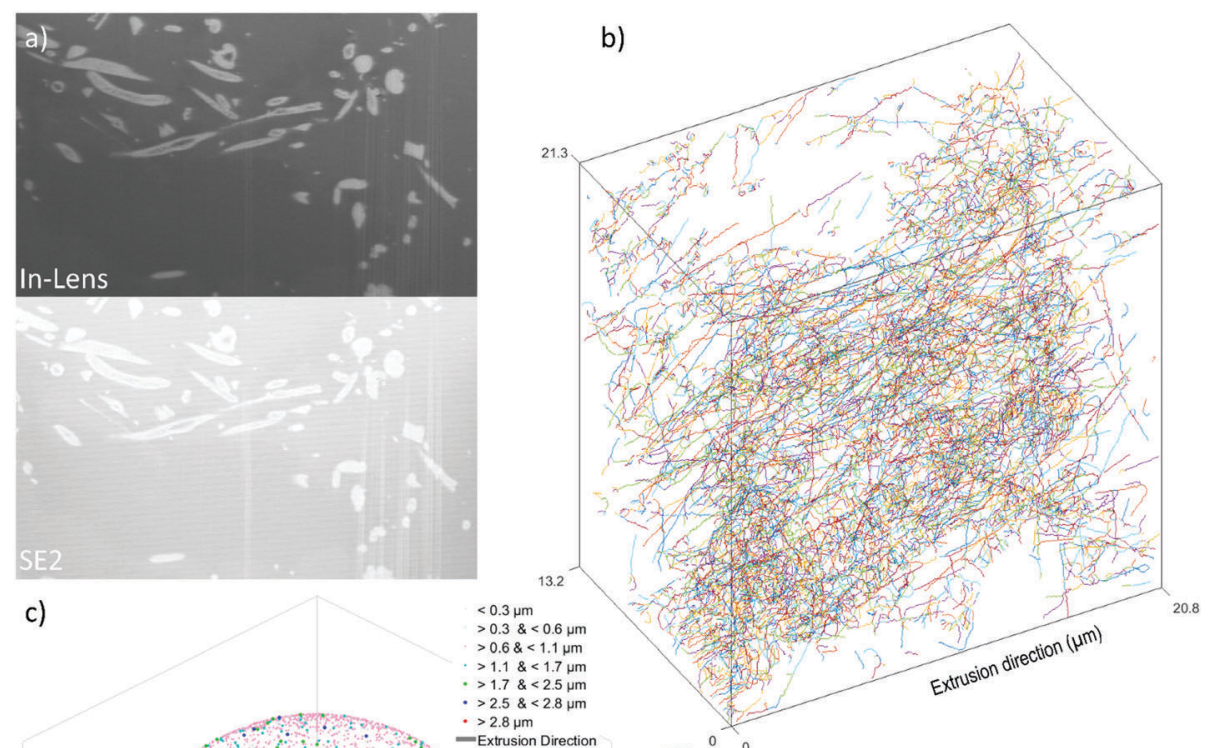

c)
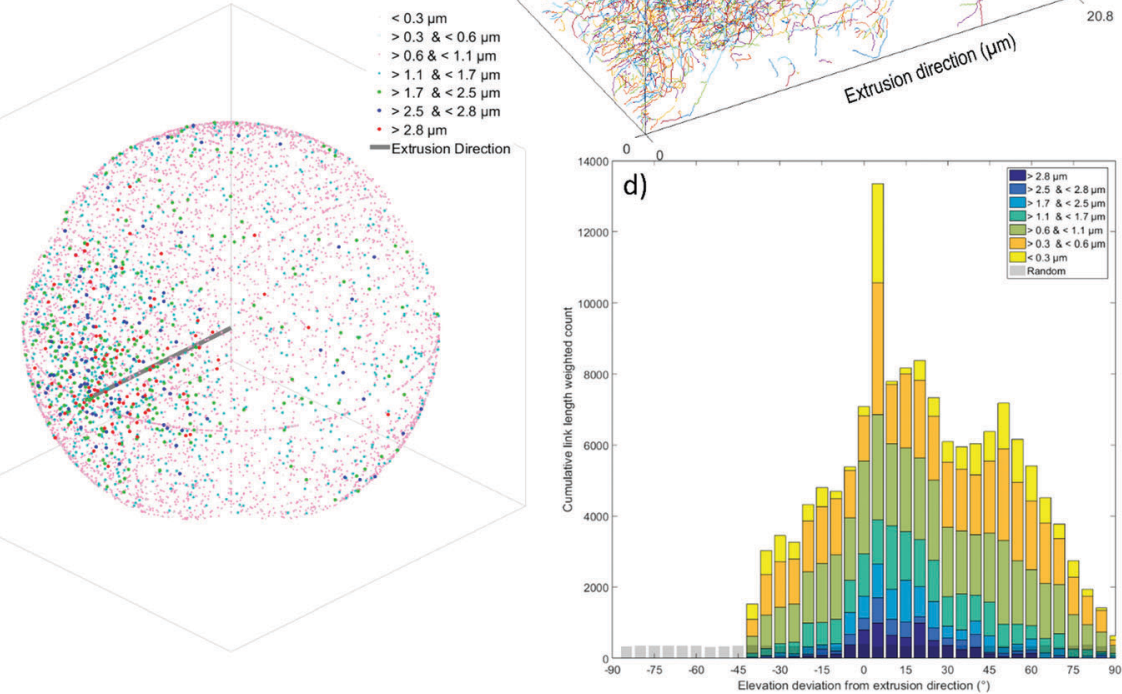

Fig. 6 (a) In-lens and SE2 detector raw image slices from the middle of the vertical axis in the volume in (b). (b) A discretized skeleton of BTNFs segmented from the PVDF. Individual fragments of the BTNFs have been assigned random colors for visualization. (c) Orientation of individual BTNF fragments plotted on a unit sphere, $b$ and $c$ are approximately aligned. (d) Stacked histograms of BTNF fragment angular (elevation) deviation from the extrusion direction as a function of fragment length. The histogram counts of elevation angle are weighted by fragment length. Note: the random distribution is on an arbitrary count scale. 
are color coded according to binned fragment lengths. This qualitatively shows that the orientation of fiber fragments shorter than approximately $1 \mu \mathrm{m}$ ( $\sim 20$ voxels in length) is randomly determined. Longer fragments however tend to be oriented close to the applied shear stress/extrusion direction (ED), and although fewer in number increasing fiber length correlates to stronger alignment.

Fig. 6d shows the distribution of fiber fragment ED angular deviation $\left( \pm 90^{\circ}\right.$ elevation angle in polar coordinates) for the same fragment length bins in Fig. 6c weighted according to the cumulative fiber fragment length. Again, the fragments shorter than $\sim 1 \mu \mathrm{m}$ reveal a widespread in elevation angle with a strong bimodal peak parallel to ED and smaller peak centered at $45^{\circ}$. It is expected that the shorter skeleton links are more likely to be associated with the aforementioned segmentation uncertainties caused by large PVDF pores and fine scale internal BTNF porosity than the longer fragments. The peaks at 0 and $45^{\circ}$ are also expected to be artificially increased due to the voxelization of the skeleton links on a rectilinear grid. In Fig. $6 \mathrm{~b}$, it is possible to see long individual fibers; however, the histogram statistically indicates that these fibers are broken into smaller fragments during skeletonization due to intersecting fibers. As observed in Fig. 6c increasing fiber fragment length strongly correlates with a narrowing angular deviation and alignment with ED.

For reference, the same number of fiber fragments (nonlength weighted) in a random distribution of angles was simulated using spherical point picking. ${ }^{51}$ The gray colored bins in the histogram show that randomly oriented fibers do not show preferred correlation to ED contrary to the BTNFs.

To better understand the effect of the processing conditions on the nanofiber alignment, the rheological behavior of the pure PVDF and BTNFs/PVDF nanohybrids was investigated. In particular, the range of interest $\left(1-100 \mathrm{~s}^{-1}\right)$ was selected to include the shear conditions that characterize the printing with the nozzles at 2.5 and $1.2 \mathrm{~mm}$ in diameter, as indicated in Fig. 7a. The two printing conditions identify a range of shear rates that spans from around 5 to $30 \mathrm{~s}^{-1}$.

The viscosity curves (Fig. 7a) indicate a shear-thinning behavior for all the samples. As expected, the profiles were progressively shifted to higher values of viscosity as the BTNF content increases, due to the flow hindering effect. ${ }^{52,53}$ Yet, the solution at the highest content of BTNFs (20 vol\%) not only exhibited the highest values of viscosity but also the most pronounced shear thinning profile. This behavior was quantitatively estimated by analyzing the flow indexes (n) via the logarithmic form of the power law. The flow index is a measure of the deviation from Newtonian behavior $(n=1)$. Plotting the experimental data, a linear trend is obtained as indicated in Fig. 7b, generally referred to as a double logarithmic power law plot. The slope of the straight line corresponds to the flow index (estimated by linear regression) that can be used to identify the flowing mechanism of a system. ${ }^{54-57}$ Any variation of the flow index within a defined range of shear rates can be associated with variation in the flowing mechanism. Fig. 7b shows the double logarithmic plot for the developed solutions, whereas in
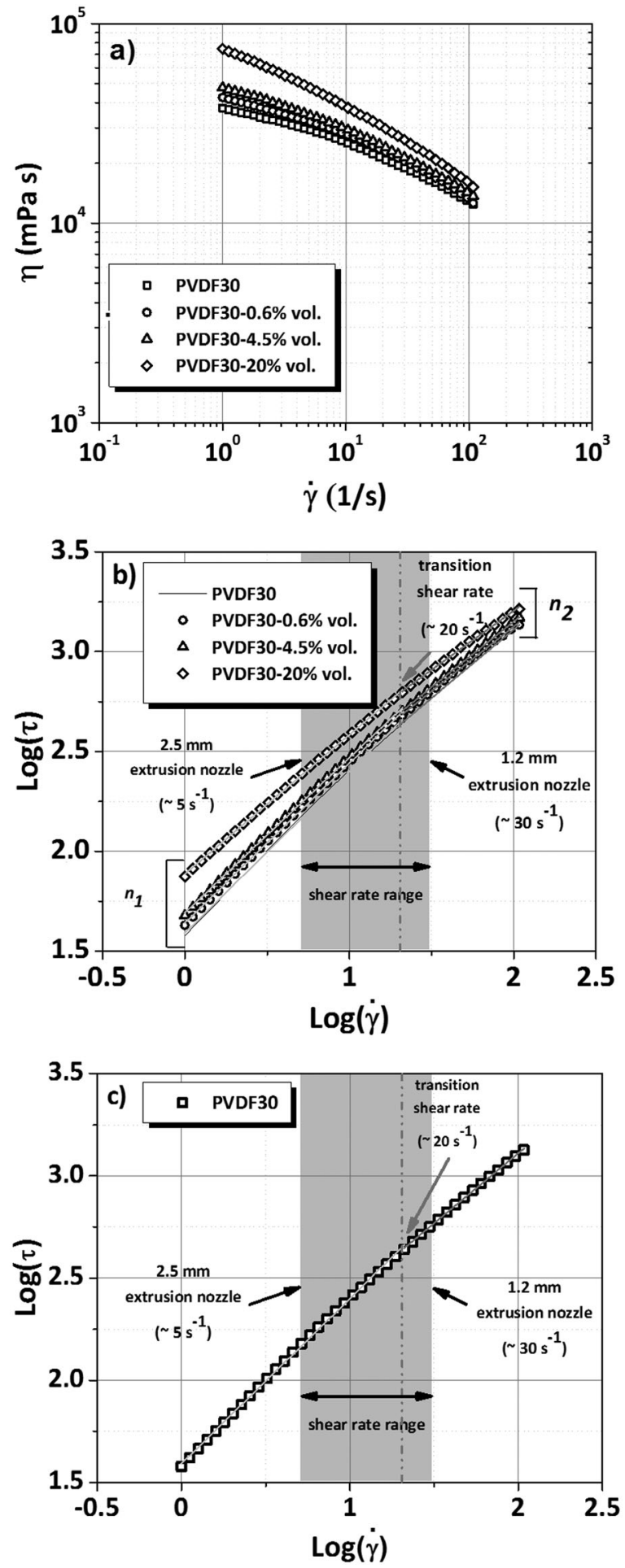

Fig. 7 (a) Viscosity plot and the corresponding (b) double logarithmic power law plot for the complete series of PVDF-based solutions showing the flow index (slope) and (c) double logarithmic power law plot for the PVDF solutions.

Table 1 the corresponding flow indexes are reported. As a consequence of the increasing BTNF loading, the values of the flow index gradually decrease, indicating an increasing shear thinning behavior $(n<1)$ (see Table 1$)$. Yet, all the 
Table 1 The flow indexes for the PVDF-based solutions

\begin{tabular}{lll}
\hline & Flow index & \\
\cline { 2 - 3 } Samples & $n_{1}\left(\dot{\gamma}<20 \mathrm{~s}^{-1}\right)$ & $n_{2}\left(\dot{\gamma}>20 \mathrm{~s}^{-1}\right)$ \\
\hline Pure PVDF & 0.81 & 0.69 \\
PVDF-BTNFs-0.6 vol\% & 0.80 & 0.67 \\
PVDF-BTNFs-4.5 vol\% & 0.78 & 0.65 \\
PVDF-BTNFs-20 vol\% & 0.70 & 0.59
\end{tabular}

samples, including the pure PVDF (see Fig. 7c), exhibit a Multi Flow Index (MFI) profile with two flow indexes associated with two distinct flowing mechanisms, both resulting in shear thinning. Interestingly for all of them, the second mechanism exhibits a more pronounced shear thinning behavior, being $n_{2}<n_{1}$. This result indicates for the flowing mechanism at higher shear rate a more pronounced deviation from Newtonian behavior likely associated with a transition in the polymer chain alignment. Interestingly, the transition is not altered by the addition of the BTNFs, since for all the PVDF samples it occurs at the same shear rate, which is around $20 \mathrm{~s}^{-1}$. This value is within the range defined by the nozzle diameters. As a result, all the PVDF samples printed using the nozzle with a diameter of $2.5 \mathrm{~mm}$ are processed at a shear rate (around $5 \mathrm{~s}^{-1}$ ) that characterizes the first mechanism of flowing. By contrast, all the PVDF samples printed using the nozzle with a diameter of $1.2 \mathrm{~mm}$ are processed at a shear rate (around $30 \mathrm{~s}^{-1}$ ) that characterizes the second mechanism of flowing.

The higher flow index in the first flowing mechanism region $\left(n_{1}\right)$ indicates a lower level of interaction among PVDF chains, likely aligned into the direction of the applied shear stress. This arrangement can favor the interaction between the BT nanofibers and the polymer chains along the direction of applied shear stress. Such an arrangement is achieved when the nozzle diameter is $2.5 \mathrm{~mm}$. By contrast, when the processing conditions exceed the identified threshold $\left(20 \mathrm{~s}^{-1}, n_{2}\right)$, the alignment might be disrupted, as indicated by a higher level of the interactions among the polymer chains $\left(n_{2}<n_{1}\right)$. As a consequence of that, the BT nanofibers are randomly distributed within the polymer matrix. This is obtained when solutions are printed using the nozzle with a diameter of $1.2 \mathrm{~mm}$.

Fig. 8a and b show the dielectric constant $(k)$ at room temperature $\left(25{ }^{\circ} \mathrm{C}\right)$ of pure PVDF and BTNFs/PVDF nanohybrids plotted against frequency for different vol\% of BTNFs in the cross-direction $\left(\mathrm{CD}, k^{\perp}\right)$ and parallel direction (PD, $k^{\|}$), respectively. The dielectric constant of all the samples decreases with increasing frequency from $100 \mathrm{~Hz}$ to $1 \mathrm{MHz}$.

Higher values observed at low frequency $(100 \mathrm{~Hz}-1 \mathrm{kHz})$ are due to the interfacial polarization. The motion of space charges in the interface zone under the applied electric field induces significant polarization, thus producing high $k$-value. By contrast, at higher frequencies the dielectric properties of materials and lower values are then observed. ${ }^{34,58}$ In all the cases, the dielectric constants of the obtained BTNFs/PVDF nanohybrids are higher than the value of the pure PVDF, but lower than pure BT nanofibers $(c a .1000)^{52}$ because of the synergy effect between the two phases. ${ }^{59}$
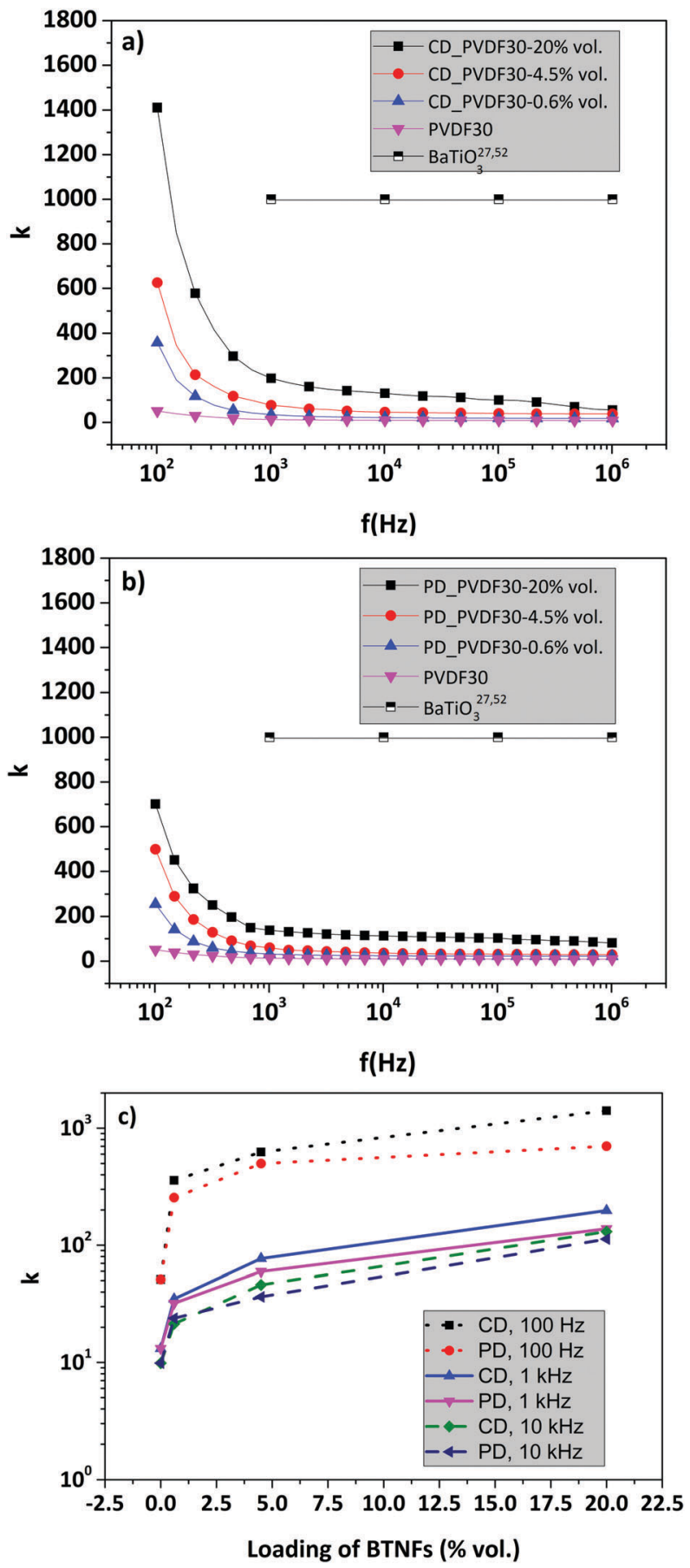

Fig. 8 Frequency dependence of the dielectric constant of (a) BTNFs/ PVDF 3D-nanohybrid dielectric materials in the cross direction (CD, $k^{\perp}$ ) and (b) in the parallel direction (PD, $k^{\|}$), and (c) dielectric constant of anisotropic BTNFs/PVDF 3D-nanohybrid dielectric materials at $100 \mathrm{~Hz}$, $1 \mathrm{kHz}$, and $10 \mathrm{kHz}$ at room temperature.

The values of $k^{\perp}$ are higher than the $k^{\|}$ones at any content of BTNFs. Specifically, in hybrid materials with $20 \mathrm{vol} \%$ of BTNFs at $1 \mathrm{kHz}$, the $k^{\perp}$ and $k^{\|}$values are 198 and 138, respectively. It can thus be concluded that the preferential nano-fiber alignment correlates strongly with the directionality of the observed dielectric constant. ${ }^{27}$ At $1 \mathrm{kHz}$, the dielectric 

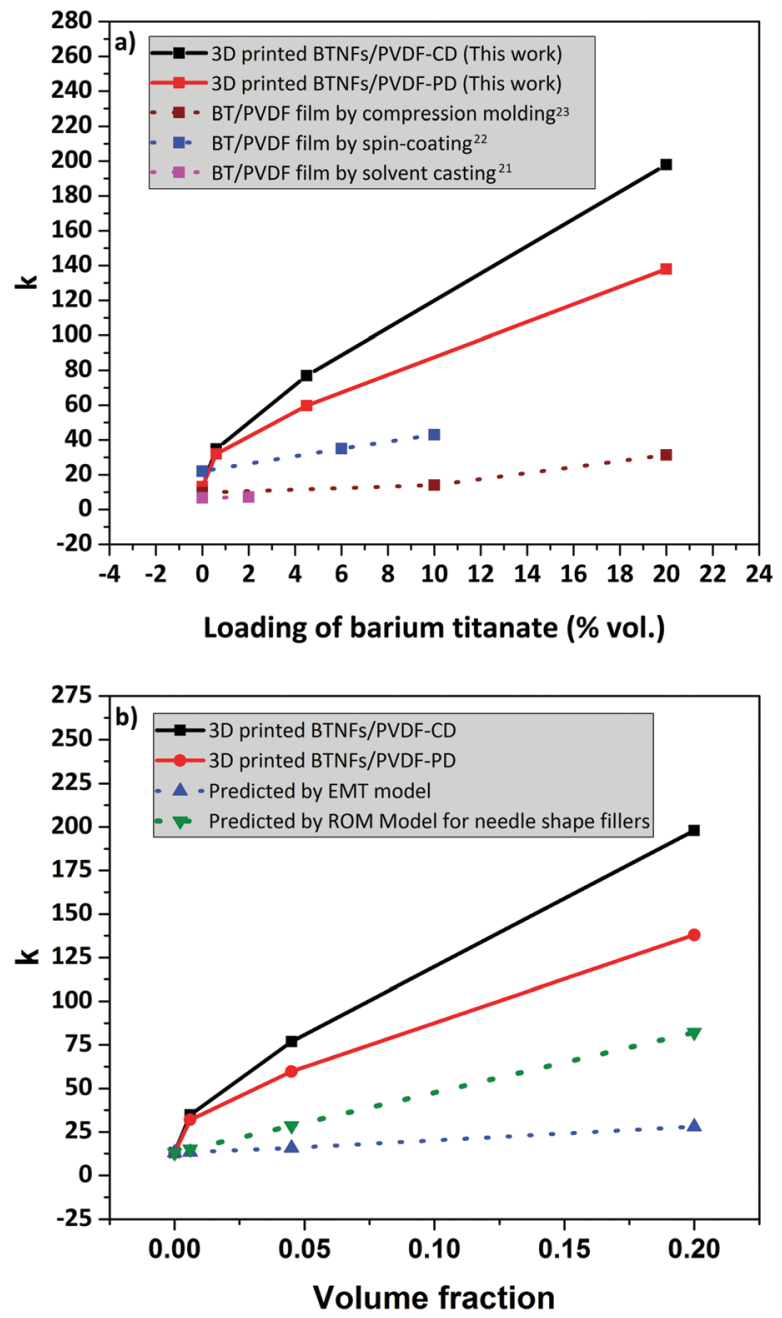

Fig. 9 (a) Dielectric constant comparison between the 3D-printed BTNFs/PVDF composites and the reported $\mathrm{PVDF} / \mathrm{BaTiO}_{3}$ composites at $1 \mathrm{kHz}$, room temperature and (b) dielectric constant at $1 \mathrm{kHz}$ from experiments vs. predicted values from model equations.

constants of hybrid samples in both the cross direction (CD) and parallel direction (PD) increase with increasing amount of BTNFs from $0.6 \mathrm{vol} \%$ to $20 \mathrm{vol} \%$ (Fig. 8c). This trend is due to the addition of a higher amount of high- $k$ component, resulting in higher- $k$ values. The highest dielectric constant $(k)$ of 198 was achieved from the sample with $20 \mathrm{vol} \%$ of BTNFs in the cross direction evaluation.

Interestingly, compared with nanohybrids prepared with different methods and containing BT nanoparticles as fillers, the BTNFs/PVDF systems herein developed show higher- $k$ values at any content of BT nanofibers. In Fig. 9a, a comparison with different BT nanoparticles/PVDF hybrids prepared via solvent casting, ${ }^{21}$ spin-coating, ${ }^{22}$ and compression molding ${ }^{23}$ is indicated. This result can be ascribed to the incorporation of high aspect ratio nanofiber fillers that determine a significant increment of the hybrid interface (Fig. 9a). ${ }^{7,17,27}$

In this work, we used effective medium theory (EMT) (eqn (2)) with fitting factor, $n=0.2, \varepsilon_{\text {filler }}=1000$ and $\varepsilon_{\text {matrix }}=13$, and the rule of mixtures (ROM) model for needle shape fillers (eqn (3)) to evaluate the $k$-values for the systems developed. The deviation of the experimental data from the models was then estimated. The results show that at any NFBT volume fraction, the experimental data deviate from the calculated $k$-values (obtained with the EMT and ROM models) (Fig. 9b). This is especially verified at the highest content (20 vol\% BTNFs), where deviations of around $80 \%$ and $50 \%$ on average are obtained for the EMT model and the ROM model, respectively. This is likely to be ascribed to the tendency of nanostructured fillers to agglomerate, which does not allow a homogeneous distribution of the nanofillers within the PVDF matrix.

\section{Conclusions}

Barium titanate nanofibers-poly(vinylidene fluoride) nanohybrid dielectric materials (BTNFs/PVDF) are prepared by the 3D-printing-FDM technique. Phase compositions, fibers alignment, and dielectric constants are achieved by embedding high aspect ratio short-nanofibers in various concentrations. BTNFs induce high anisotropic behavior in the hybrids. Alignment of BTNFs is triggered by a delicate interplay of viscoelastic effects at the printing throughout internal and external frictions within the hybrid and at the wall of the extruders at different flow rates. High enhancement of dielectric properties of more than 1 order of magnitude with respect to the pure PVDF can be achieved. Specifically, the BTNFs/PVDF 3D-nanohybrid dielectric material, with 20 vol\% BTNFs, shows a dielectric constant in the cross-direction of around 200 at a frequency of $1 \mathrm{kHz}$ at room temperature, $v s .13$ of the pristine PVDF materials. This enhancement can be associated with an extensive hybrid interface developed by the use of high aspect ratio 1D nanofillers. The anisotropic flexible 3D nano-hybrid composite with high- $k$ constants is attractive for complex-shaped embedded capacitors and electric energy storage devices. We here demonstrate that this concept enables novel functionalities in dielectric components which could be applied for complex-shaped embedded capacitor applications. We believe that the FDM technique provides great potential for developing high- $k$ ferroelectric ceramic/polymer composites with controllable anisotropic properties.

\section{Conflicts of interest}

There are no conflicts of interest to declare.

\section{Acknowledgements}

The authors would like to thank the Royal Golden Jubilee PhD Program (RGJ-PhD Program), Thailand (grant no. PHD/0116/2557) for financial support and Department of Energy Conversion and Storage, DTU Energy, Technical University of Denmark for supporting the research. JRB would like to thank Peter Stanley Jørgensen for many useful image analysis and principle component analysis discussions. 


\section{References}

1 N. R. Alluri, B. Saravanakumar and S. J. Kim, ACS Appl. Mater. Interfaces, 2015, 7, 9831-9840.

2 X. Ni, F. Wang, A. Lin, Q. Xu, Z. Yang and Y. Qin, Sci. Adv. Mater., 2013, 5, 1781-1787.

3 S. H. Shin, Y. H. Kim, M. H. Lee, J. Y. Jung and J. Nah, ACS Nano, 2014, 8, 2766-2773.

4 Y. Zhang, Y. Zhang, X. Xue, C. Cui, B. He, Y. Nie, P. Deng and Z. L. Wang, Nanotechnology, 2014, 25, 105401.

5 Y. Bai, Z. Y. Cheng, V. Bharti, H. S. Xu and Q. M. Zhang, Appl. Phys. Lett., 2000, 76, 3804-3806.

6 H. Hammami, M. Arous, M. Lagache and A. Kallel, J. Alloys Compd., 2007, 430, 1-8.

7 L. Nicole, C. Laberty-Robert, L. Rozes and C. Sanchez, Nanoscale, 2014, 6, 6267-6292.

8 D. L. Kerbow and C. A. Sperati, Wiley Database of Polymer Properties, 1999.

9 B. Chu, X. Zhou, K. Ren, B. Neese, M. Lin, Q. Wang, F. Bauer and Q. Zhang, Science, 2006, 313, 334-336.

10 Y. Kobayashi, T. Tanase, T. Tabata, T. Miwa and M. Konno, J. Eur. Ceram. Soc., 2008, 28, 117-122.

11 Y. Li, K. Moon and C. P. Wong, Science, 2005, 308, 1419-1420.

12 S. Bodkhe, G. Turcot, F. P. Gosselin and D. Therriault, ACS Appl. Mater. Interfaces, 2017, 24, 20833-20842.

13 M. S. Gaur, P. K. Singh, A. Ali and R. Singh, J. Therm. Anal. Calorim., 2014, 3, 1407-1417.

14 H. Luo, J. Roscow, X. Zhou, S. Chen, X. Han, K. Zhou, D. Zhang and C. R. Bowen, J. Mater. Chem. A, 2017, 5, 7091-7102.

15 H. Luo, D. Zhag, C. Jiang, X. Yuan, C. Chen and K. Zhou, ACS Appl. Mater. Interfaces, 2015, 7, 8061-8069.

16 H. Tang, Y. Lin and H. A. Sodano, Adv. Energy Mater., 2013, 3, 451-456.

17 D. Marani, A. D’Epifanio, E. Travers, M. Miyayama and S. Licoccia, Chem. Mater., 2010, 22, 1126-1133.

18 W. S. Yun, J. J. Urban, Q. Gu and H. Park, Nano Lett., 2002, 2, 447-450.

19 J. Yuh, J. C. Nino and W. M. Sigmund, Mater. Lett., 2005, 59, 3645-3647.

20 J. J. Urban, J. E. Spanier, L. Ouyang, W. S. Yun and H. Park, Adv. Mater., 2003, 15, 423-426.

21 R. H. Upadhyay and R. R. Deshmukh, J. Electrost., 2013, 71, 945-950.

22 Y. X. Li, J. L. Xie, Z. M. Chu, X. S. Wang and X. Yao, Adv. Mater. Res., 2014, 1, 365-369.

23 Y. Li, S. C. Tjong and R. Li, eXPRESS Polym. Lett., 2011, 5, 526-534.

24 K. Kim, W. Zhu, X. Qu, C. Aaronson, W. R. McCall, S. Chen and D. J. Sirbuly, ACS Nano, 2014, 8, 9799-9806.

25 F. Castles, D. Isakov, A. Lui, Q. Lei, C. Dancer, Y. Wang, J. Janurudin, S. Speller, C. Grovenor and P. S. Grant, Sci. Rep., 2016, 6, 22714.

26 K. Sun, T. S. Wei, B. Y. Ahn, J. Y. Seo, S. J. Dillon and J. A. Lewis, Adv. Mater., 2013, 25, 4539-4543.
27 I. Krakovský and V. Myroshnychenko, J. Appl. Phys., 2002, 92, 6743-6748.

28 J. Yuh, L. Perez, W. M. Sigmund and J. C. Nino, J. Sol-Gel Sci. Technol., 2007, 42, 323-329.

29 P. S. Jørgensen, S. L. Ebbehøj and A. Hauch, J. Power Sources, 2015, 279, 686-693.

30 P. S. Jørgensen, K. V. Hansen, R. Larsen and J. R. Bowen, Ultramicroscopy, 2010, 110, 216-228.

31 GitHub, Inc., (US), https://github.com/phi-max/skeleton3dmatlab, accessed September, 2017.

32 M. Kerschnitzki, P. Kollmannsberger, M. Burghammer, G. N. Duda, R. Weinkamer, W. Wagermaier and P. Fratzl, J. Bone Miner. Res., 2013, 28, 1837-1845.

33 GitHub, Inc., (US), https:/github.com/phi-max/skel2graph3dmatlab, accessed September, 2017.

34 P. I. Devi and K. Ramachandran, J. Exp. Nanosci., 2011, 6, 281-293.

35 R. Yang, J. Qu., T. Marinis and C. P. Wong, IEEE Trans. Adv. Packag., 2000, 23, 680-683.

36 W. S. Chen and M. Y. Hsieh, Ceram. Int., 2017, 43, S343-S350.

37 S. Kumar, G. L. Messing and W. B. White, J. Am. Ceram. Soc., 1993, 76, 617-624.

38 S. Maensiri, W. Nuansing, J. Klinkaewnarong, P. Laokul and J. Khemprasit, J. Colloid Interface Sci., 2006, 297, 578-583.

39 H. Kozuka and A. Higuchi, J. Mater. Res., 2001, 16, 3116-3123.

40 D. Y. Lee, M. H. Lee, N. I. Cho, B. Y. Kim and Y. J. Oh, Met. Mater. Int., 2010, 16, 453-457.

41 Z. L. Wang and J. Song, Science, 2006, 312, 242-246.

42 B. Ding, C. Li, S. Fujita and S. Shiratori, Colloids Surf., A, 2006, 284, 257-262.

43 L. Li, P. Zhang, J. Liang and S. Guo, Ceram. Int., 2010, 36, 589-594.

44 D. Marani, R. H. Silva, A. Dankeaw, K. Norrman, R. M. L. Werchmeister, D. Ippolito, M. Gudik-Sørensen, K. K. Hansen and V. Esposito, New J. Chem., 2017, 41, 3466-3472.

45 L. Yin, J. Niu, Z. Shen, Y. Bao and S. Ding, Mater. Lett., 2011, 65, 3131-3133.

46 R. Singh, J. Kumar, R. K. Singh, A. Kaur, R. D. P. Sinha and N. P. Gupta, Polymer, 2006, 47, 5919-5928.

47 S. Rajendran, P. Sivakumar and R. S. Babu, J. Power Sources, 2007, 164, 815-821.

48 S. Yu, W. Zheng, W. Yu, Y. Zhang, Q. Jiang and Z. Zhao, Macromolecules, 2009, 42, 8870-8874.

49 Z. Li, X. Zhang and G. Li, Phys. Chem. Chem. Phys., 2014, 16, 5475-5479.

50 J. A. Lewis, J. E. Smay, J. Stuecker and J. Cesarano, J. Am. Ceram. Soc., 2006, 89, 3599-3609.

51 Wolfram Research, Inc., http://mathworld.wolfram.com/ Sphere PointPicking.html, accessed September, 2017.

52 D. Wu, J. Wang, M. Zhang and W. Zhou, Ind. Eng. Chem. Res., 2012, 51, 6705-6713.

53 S. Vidhate, A. Shaito, J. Chung and N. A. D'Souza, J. Appl. Polym. Sci., 2009, 112, 254-260. 
54 D. Marani, J. Hjelm and M. Wandel, J. Eur. Ceram. Soc., 2014, 34, 695-702.

55 D. Marani, B. R. Sudireddy, J. J. Bentzen, P. S. Jørgensen and R. Kiebach, J. Eur. Ceram. Soc., 2015, 35, 2823-2832.

56 D. Marani, C. Gadea, J. Hjelm, P. Hjalmarsson, M. Wandel and R. Kiebach, J. Eur. Ceram. Soc., 2015, 35, 1495-1504.
57 D. Marani, V. Esposito, B. R. Sudireddy, J. J. Bentzen, P. S. Jørgensen, F. Teocoli and R. Kiebach, Ceram. Int., 2017, 43, 5647-5653.

58 M. Benlahrache, S. Barama, N. Benhamla and S. Achour, Mater. Sci. Semicond. Process., 2006, 9, 1115-1118.

59 G. Hu, F. Gao, J. Kong, S. Yang, Q. Zhang, Z. Liu, Y. Zhang and H. Sun, J. Alloys Compd., 2015, 619, 686-692. 